\title{
Successor states in a four-state ambiguous figure
}

\author{
GREGORY BURTON \\ Seton Hall University, South Orange, NJ
}

\begin{abstract}
The satiation theory of ambiguous figures holds that interpretation shifts are caused by fatigue of neural arrangements responsible for the prevailing interpretation. A four-state multistable figure is introduced, in which two depicted cubes can be seen as connected or unconnected and as facing up or facing down. Observers viewed the figure for $4 \mathrm{~min}$. When descriptive labels were used for the interpretations, shifts to interpretations that shared neither dimension were significantly more frequent than shifts that conserved orientation or connection/disconnection. However, all types of transitions were equally likely when arbitrary letter codes were used, implying that the putatively fatigued assemblies can be dedicated to whole figures or to their characteristics, depending on observer expectations.
\end{abstract}

One of the predominant contemporary approaches to ambiguous figure reversals (see, e.g., Long, Toppino, \& Mondin, 1992) is the satiation theory, which dates back to Wolfgang Köhler (1940; for refinements, see e.g., Attneave, 1971; Hochberg, 1950; Hock, Schöner, \& Voss, 1997). It presumes that partially distinct neural pathways are activated by rival interpretations of a bistable figure and that perception shifts when the prevailing complex of activation becomes fatigued. Köhler tentatively suggested that the buildup of some substance or quality among communicating neurons caused both the fatigue itself and the commonly observed decrease in the time necessary to reach fatigue with each cycle, a process that Attneave (1971) compared to a multistable electrical circuit. Gomez and colleagues (Gomez, Argandoña, Solier, Angulo, \& Vázquez, 1995) offered a more mathematical model that predicted a sigmoid time course for the maintenance of any neural representation in the face of a competing interpretation.

There are several other major theories about reversible figures, and some share with the fatigue hypothesis the framework of a rivalry between two interpretations. For example, Miller et al. (2000) reported a converging series of experiments linking reversible figures with binocular rivalry and attributed both phenomena to a rivalry between brain hemispheres. These speculations naturally lead one to wonder about ambiguous figures with more than two interpretations. Such multistable figures have been discussed; for instance, Attneave (1968) and Bucher and Palmer (1985) have considered the ambiguous orientation of triangles, and Kubovy (1994) has analyzed the unstable interpretation of dot lattices. Fisher (1967) published

Portions of this research were presented at the February 1998 meeting of the Eastern Psychological Association in Boston. I would like to acknowledge the assistance of Giselle Lupiañez, Zalman Vaksman, and Violeta Gulevska in conducting the experiments, as well as Julian Hochberg and an anonymous reviewer for helpful comments. Correspondence concerning this article should be addressed to G. Burton, Seton Hall University, South Orange, NJ 07079 (e-mail: burtongr@ shu.edu). drawings that could be seen in four ${ }^{1}$ ways, and Broerse, $\mathrm{Li}$, and Ashton (1994) investigated a three-loop figure that could be seen as flat or as one of two arrangements in depth. However, the satiation hypothesis has rarely been applied to ambiguous figures with more than two interpretations. Attneave (1971) noted that the perceived orientation of triangles changes over time, but subsequent analysis of triangles (Palmer, 1980; Palmer \& Bucher, 1981) has focused on constraints on the original interpretation, rather than on subsequent shifts.

If more than two perspectives are possible, will the perceived perspective, when it shifts from the satiated prevailing interpretation to another from the set of possible interpretations, shift to a predictable successor or simply to the interpretation least recently or least frequently activated? Does the satiation hypothesis require that perception rotate among all the possible states?

Figure 1 shows a new ambiguous figure that may be suitable for applying the satiation hypothesis to multistable figures. I will refer to the figure as a double-decker Necker; it consists of two Necker cubes in which the lower right diagonal of the upper figure is colinear with the upper left diagonal of the lower figure. The figure can be seen as two cubes connected at the collinear diagonal or as two cubes separated by a narrow channel; each of these interpretations can be seen as facing toward or away from the observer (see Figure 2). I believe this figure to be useful, not only because of the four possible interpretations, but also because the interpretations are nested in a $2 \times 2$ relationship, allowing some preliminary investigation of the possibility that orthogonal characteristics of the interpretations can "fatigue" independently of the entire interpretation. For example, if the current perception is the one depicted at the upper left in Figure 2, can the apparent separation or the "up-facing-ness" fatigue independently, or is there simply a fatigue of "up-facing separated"

Once an observer has satiated one of the four states depicted in Figure 2, three new interpretations are possible. Will the successors be predictable? The present experiment was designed to address this preliminary question. There 


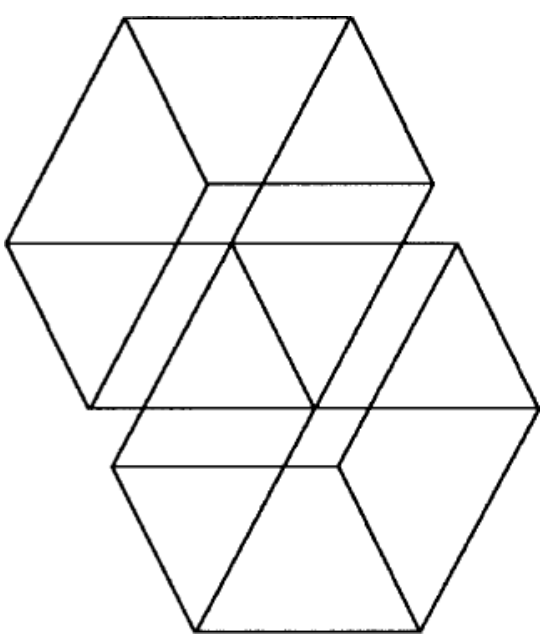

Figure 1. The double-decker Necker cube. The figure was presented to observers on a circular background.

are at least two categories of possible shift patterns. Three possible patterns involve geometrical aspects of the interpretations; shifts from interpretations that share an orientation or their separation/connection characteristic may be favored over shifts that conserve neither characteristic. An analysis of variance (ANOVA) will test whether these three possible shifts are equally represented. Two possible predictions involve temporal aspects of the shifting interpretations. The observer might shift to the interpretation least recently activated; if this is the case, the absolute number of activations of each interpretation should be roughly equal. Or the successor state may be the one with the lowest cumulative activation (i.e., with the least fatigue "buildup" over the course of observation). If this is the case, the total time spent on the four interpretations should be roughly equal.

\section{EXPERIMENT 1 Meaningful Labels for Interpretation}

\section{Method}

Subjects. Twenty-seven undergraduates (11 males and $15 \mathrm{fe}-$ males; the gender of 2 subject was not recorded) took part for extra credit in an introductory level course; the data for 2 subjects were not recorded, owing to computer problems. These observers also took part in an unrelated visual experiment during the same session; the order of the two experiments was counterbalanced.

On the basis of a preliminary observation of the figure (see below), the observers were classified according to how many interpretations of the figure they could see before being prompted. Five observers could report only one interpretation, 11 and 6 could report two and three interpretations, respectively, and 3 observers could report all interpretations without being prompted.

Materials. The stimuli reproduced as Figures 1 and 2 were printed in black on white copier paper; the longest axis of the figure (from upper left to lower right) was $12.2 \mathrm{~cm}$. Figure 1 was attached to a circular piece of cardboard. Materials also included a copy of the reversible spiral figure and a pair of small paper boxes (side length, $2.5 \mathrm{~cm}$ ) used for portraying different arrangements of two cubes.
The experimenter used a computer program on a IBM-compatible computer to time the duration of different interpretations of the figure.

Procedure. The observers were first introduced to the concept of ambiguous figures, using the familiar spiral figure; most reported having encountered ambiguous figures before. Then they were shown the double-decker Necker printed on the circular cardboard, and their original interpretations were recorded. The observers were invited to report other interpretations of the figure, and the number of interpretations they could generate was recorded; afterward, they were shown any of the four major interpretations they had not yet seen. Figure 2 and the paper cubes were employed at this point.

Next, the observers were coached in and tested on a code for reporting their current interpretations. The code was modified after the first 2 observers were tested when it became clear that the first version was excessively difficult. The final code comprised all combinations of stacked (if the cubes appeared to be separated by a narrow space) versus diamond (if the tops of the cubes appeared to be two diamonds touching at a corner) and of top (if the uppermost Yvertex would be visible if the cubes were solid) versus bottom (the lower Y-vertex would be visible). Perusal of Figure 2 may suggest that the interpretations labeled top appear to face the bottom; however, pilot experimentation revealed great difficulty in stating unambiguously which face of the line figure (Figure 1) was to be assessed for its direction. The Y-vertices were lightly circled on the version of Figure 1 to facilitate identification of the desired features of the figure. Before moving to the next stage, the observers were required to correctly name all four interpretations, using the code three times in a row.

In the main portion of the experiment, the double-decker figure, oriented as in Figure 1, was viewed for $4 \mathrm{~min},{ }^{2}$ and any shifts were reported, using the code; the experimenter used a computer program to record the shifting interpretations and the time spent at each interpre-
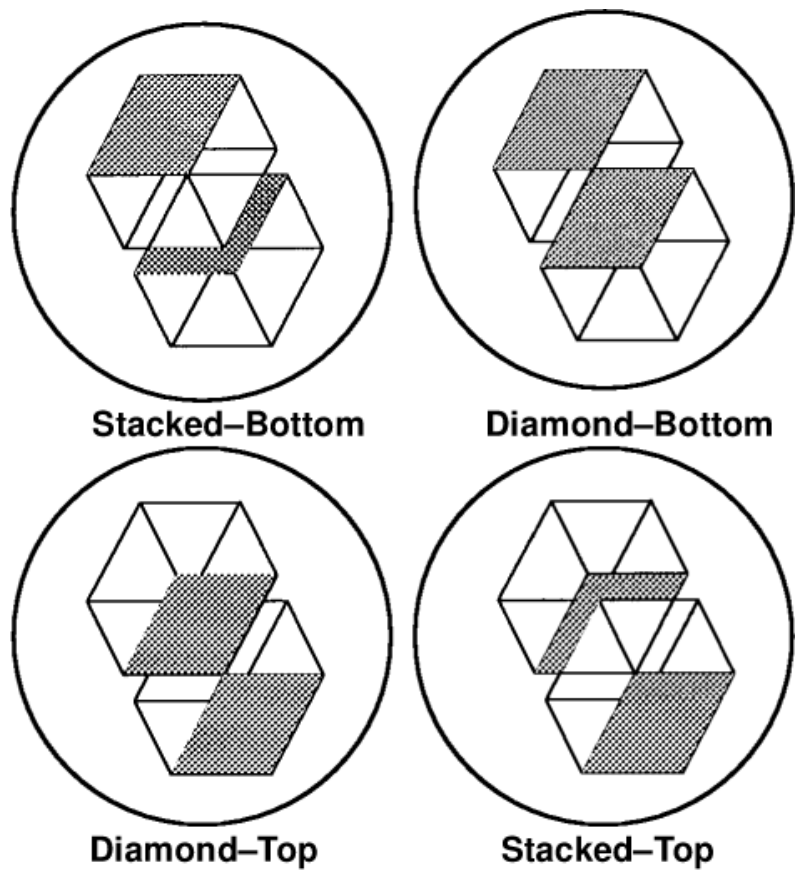

Figure 2. This figure was used to show observers four possible interpretations of Figure 1 and to practice the code. Top and Bottom refer to which of the simple Y-junctions would be visible if the closest face of each cube was opaque, as depicted in this figure. The code for each interpretation is shown here but was not shown to the subjects in the experiment. 
tation. The observers were cautioned that they were under no obligation to "use" all four interpretations or to shift a certain number of times.

\section{Results and Discussion}

The most common first interpretation was stackedtop (lower right on Figure 2; first interpretation for $12 \mathrm{ob}$ servers), followed by stacked-bottom (upper left; 7 observers), diamond-bottom (upper right; 5 observers) and diamond-top (lower left; 0 observers). A chi-square test found that the pattern of first interpretations was significantly different from chance $\left[\chi^{2}(3)=12.4, p<.01\right]$. Not only did no observer see the diamond-top interpretation first, but only 9 saw it at all without being prompted.

The remaining analyses centered on the predictability of shifts. Palmer and Bucher (1981) asserted that one interpretation of an ambiguous figure is unlikely to be reactivated before the remaining interpretations have dominated, a pattern they refer to as sampling without replacement. However, this assertion was premised on the idea that each interpretation has an equivalent threshold for activation, which may be true of the orientation of triangles but seems contraindicated by the result above for the doubledecker Necker. So it is important to check for sampling without replacement for this figure. If the interpretation least recently activated is likely to be the successor, the number of visits to each interpretation should balance out; each would be activated roughly every fourth shift.

In contradiction to this hypothesis, the absolute number of times each interpretation was seen was significantly different $[F(3,63)=4.7, p<.01]$, with the diamond-top interpretation that was never the first interpretation being seen least often. A Tukey post hoc test (.01) showed that the figure was classified as diamond-top significantly fewer times $(M=3.5)$ than as stacked-top (5.8) or stackedbottom (5.6); no other mean comparisons were significant (for diamond-bottom, $M=4.8$ ). The subject variable of number of unprompted observations was included in the ANOVA, but neither it $[F(3,21)<1.0)$ nor the interaction $[F(9,63)<1.0]$ was significant.

It is, of course, possible that the absolute number of activations of one interpretation is less important than the cumulative time spent at that interpretation, in which case I would expect that the times would be roughly equal for the four states. In fact, however, the diamond-top interpretation was also seen for the shortest amount of time, $34 \mathrm{sec}$ on average, out of the roughly $240 \mathrm{sec}$ available. A one-way ANOVA showed the variable of interpretation to be significant $[F(3,72)=5.9, p<.01]$, and a Tukey test found that diamond-top was seen for significantly $(p<.01)$ less time $(M=34.3 \mathrm{sec})$ than were the other three interpretations, which were seen for between 63 and $65 \mathrm{sec}$ each and were not significantly different. Because each observer had $240 \mathrm{sec}$ to view the figure, the between-subjects variable of Number of unprompted observations was not incorporated into the analysis of time. The difficulty of seeing one of the interpretations probably reflects unique aspects of the drawing, and not anything of great psychological import, but its neglect by the observers shows that they did not simply shift to the least often seen interpretation. Fisher (1967) described a technique in which the ambiguity of a figure could be statistically established; it involves presenting a systematic series of versions of the figure that shade from a version favoring one interpretation to a version favoring the other. It is not clear that Fisher's technique could be modified for a multistable figure, and in fact, Fisher does not employ the technique when investigating his own three- (Fisher, 1968) and four-interpretation (Fisher, 1967) figures.

The two analyses just described belie the notion that the four interpretations of the figure are equal and that the successor state when one fatigues can be predicted simply from temporal variables. Subsequent analyses considered specific aspects of the figure. Each transition from one interpretation to another was coded as sharing orientation (e.g., from diamond-top to stacked-top), sharing shape (e.g., from diamond-top to diamond-bottom), or sharing nothing (e.g., from diamond-top to stacked-bottom). Shifts that were interrupted by confusion or an interpretation different from the main ones were counted as though uninterrupted. Note that a shift from the current interpretation could be to any of the three categories, and thus the number of shifts of each type should have been roughly equal if shifting was random. ${ }^{3}$

Figure 3 depicts the mean frequency of each transition type. Nothing-shared shifts were the most likely (occurring 7.3 times, on average, per observer), and a two-way mixed design ANOVA found the transition category to be a significant variable $[F(2,42)=4.3, p<.05]$. Each observer shifted 5.4 times, on average, to an interpretation



Figure 3. The mean number of shifts in Experiment 1 that conserved orientation (e.g., diamond-top to stacked-top), conserved separation (e.g., diamond-top to diamond-bottom), or changed both (e.g., diamond-top to stacked-bottom). Separate bars are shown for the observers who, without being prompted saw only one, two, three, or all of the interpretations at the beginning of the experiment. 
sharing orientation and 5.8 times to one sharing shape; despite the significant variable, shifts of all types were quite common. Number of unprompted interpretations was not significant $[F(3,21)<1]$, nor did these two variables interact significantly $[F(6,42)<1]$.

The satiation hypothesis presumes that shifting from one to another interpretation of an ambiguous stimulus reflects the fatigue of neural arrangements responsible for visual perception. When bistable figures are considered, the purported fatigue can be conceived of in at least two ways. To take the conventional Necker cube as an example, the neural assemblies in question might represent up-facing cube and down-facing cube; such a holistic approach seems most consistent with the Gestalt psychology origins of the satiation hypothesis (Köhler, 1940). However, perceptual research since midcentury has aggressively challenged the assertion that the perception of a figure is driven by holistic considerations (Hochberg, 1998); such phenomena as the coherent perception of impossible figures when physically possible interpretations are also available and the influence of the specifically attended locus on the overall perception are among those that undermine the importance of the holistic impression of a figure. Although the notion of piecemeal perception generally concerns parts of the figure, another approach that challenges holistic perception is one that emphasizes figural qualities. Applying this perspective to the doubledecker Necker, it is also possible that the assemblies that fatigue simply represent up-facing and down-facing. There could be a different assembly responsible for the observer's perception of "cubism," and although this assembly is presumably just as vulnerable to fatigue, its fatigue has no influence, because there is no other state to which to shift. As long as there are two stable states of an ambiguous percept, the shift of interpretation is itself ambiguous and may reflect fatigue of the entire figure or of just the variable feature of that figure. ${ }^{4}$ Hochberg and Peterson (1987) are among the researchers who have concluded that fatigue of the Necker cube is piecemeal, rather than for a coherent interpretation.

The tendency in Experiment 1 for interpretation shifts to change both qualities, the apparent orientation and the separation status, implies that these qualities are the entities that fatigue, rather than a coherent image or interpretation of the figure. However, a different interpretation of Experiment 1 would be that the dissociability of the orientation and the separation qualities does not reflect neural organization, but only the hierarchical nature of the response options. 5 The requirement that the observers use the $2 \times 2$ terminology to report their interpretations may have induced an expectation that the two dimensions should be considered separately. Alternately, the observers may have experienced response fatigue entirely separate from perceptual fatigue, leading to a lower threshold for reporting interpretations that used less fatigued responses. Such a response fatigue may be analogous to the phenomenon of inhibition of return (e.g., Taylor \& Klein, 2000), often interpreted as a motor suppression of eye movements to previously scanned (and thus, generally redundant) loca- tions (note that Taylor \& Klein, 2000, and some others believe that the category of inhibition of return subsumes more than one phenomenon, not all of which involve response suppression).

To test whether the interpretation pattern in Experiment 1 reflects perceptual organization or response organization, observers were trained in Experiment 2 to use an arbitrary A-B-C-D response code that did not include descriptive labels or any suggestion of a $2 \times 2$ organization.

\section{EXPERIMENT 2 Arbitrary labels for identification}

\section{Method}

Subjects. Twenty-five undergraduates (7 males and 18 females) took part for extra credit in an introductory level course; 1 female observer failed to show up for the second session, and thus no data were collected.

Materials. The same materials were used as those in Experiment 1 . In addition, the four shaded figures that are represented as Figure 2 were separated and attached to an open manila folder above the code letters of A, B, C, or D for the four interpretations of the figure. Each possible combination of code letter and interpretation was used for 1 participant; the participant who dropped out was replaced by one trained in the same code. A study sheet depicting the same code was prepared for each observer. There was also a randomly ordered stack of five copies each of larger $(8.5 \times 11)$ shaded versions of the figure; these were shaded in a different manner in order to reduce the observer's dependency on any one particular style of shading.

Procedure. To facilitate the learning of the arbitrary code, the experiment was split into two sessions. During the first session, which lasted less than $15 \mathrm{~min}$, the observer was introduced to the concept of ambiguous figures using the spiral figure, and then was shown Figure 1. As in the first experiment, they were prompted for their interpretations; however, we did not use any terminology related to the labels from the first experiment, such as diamond, stacked, diagonal, or horizontal. Once all four interpretations had been reported by the observer or described by the experimenter, the manila folder was opened, and the A-B-C-D code was described to the observer. To test mastery of the code, the observer was shown sheets from the stack described above and was invited to identify them by code letter. Once the observer was ready to close the folder, the testing process continued until the observer had gone through the stack without an error (i.e., five repetitions of each code).

The second session took place from 1 to 12 days after the first, depending on the observer's schedule. First, the observer was tested on the practice stack, in the same manner and with the same criterion for completion as at the end of the first session. Then the experiment proceeded to a data collection phase like that in Experiment 1, with the exception that only the letters A, B, C, and D were used to identify the figures. The collection period was also expanded to $5 \mathrm{~min}$ in order to provide more data; none of the following analyses would be materially different if only the first 4 min were analyzed.

\section{Results and Discussion}

Unlike in Experiment 1, the most common first interpretation was diamond-bottom (10 observers), followed closely by stacked-top (8 observers) and stacked-bottom (7 observers). As in Experiment 1, no observer reported diamond-top first. A chi-square test found that the pattern of first interpretations was significantly different from chance $\left[\chi^{2}(3)=9.1, p<.05\right]$. As in Experiment 1, the absolute number of visits to each interpretation was sig- 
nificantly different $[F(3,69)=18.5, p<.001]$ as was the time spent on each interpretation $[F(3,69)=11.8, p<$ $.001]$. The diamond-top interpretation was again visited the least and for the least time (the number of visits was 13.0 for stacked-bottom, 14.6 for stacked-top, 12.4 for diamond-top, 12.4 for diamond-bottom, and 5.7 for diamond-top; time spent was $88.9 \mathrm{sec}$ for stacked-bottom, $83.8 \mathrm{sec}$ for stacked-top, $92.2 \mathrm{sec}$ for diamond-bottom, and $31.5 \mathrm{sec}$ for diamond-top), confirming the pattern seen when meaningful labels were used. However, one difference from Experiment 1 is that the number of visits was about twice as high; even if only the first $4 \mathrm{~min}$ of data are analyzed, the visits range from 4.5 to 11.9 , as compared with 3.5-5.8 for Experiment 1. However arbitrary, the letter labels may have been easier to say; the practice may also have facilitated the reporting of changes and led to more changes being reported overall.

Note that the interpretations were not different in their popularity if identified by letter [time, $F(3,69)=0.35$; visits, $F(3,69)=0.18)$; this is a useful confirmation that the observers preferred particular visual interpretations of the figure, rather than particular phrases or actions for reporting.

The most important analysis for Experiment 2 is whether the pattern of shifts corresponded to Experiment 1 even when the observers did not use meaningful names for the figure interpretations. The shifts that changed both dimensions of the interpretation, shape and orientation, were again the most common (15.8), but only marginally so, as compared with 15.0 for shifts conserving shape and 13.9 for shifts conserving orientation. Unlike the case for Experiment 1, this pattern was not significantly different $[F(2,46)=0.67]$; there was also no significant preference for shifting among any pairs of letters $[F(5,115)=0.43]$.

On the basis of these results, the preference for shifting from the current interpretation to one sharing neither shape nor orientation seems to be dependent on labels that suggest those two dimensions. All types of shifts are equally popular when arbitrary labels are employed that do not suggest pairings of the interpretations. However, the pattern of which interpretations were most popular was the same regardless of the response format.

\section{GENERAL DISCUSSION}

Although multistablefigures have been known for some time (e.g., Attneave, 1968; Fisher, 1968), some of the more popular theories for explaining reversible figures seem to be limited to figures that have 2 interpretations. A doubledecker Necker cube was introduced in this project as a useful tool for expanding or challenging these frameworks. I consider the major finding of both experiments to be the fact that it is quite possible for observers to rotate among more than 2 interpretations, a finding that is hard to reconcile with the idea that different hemispheres adopt the different interpretations (e.g., Miller et al., 2000). These results suggest the question of how many independent neural assemblies can operate at one time. Warren and Gregory (1958) reported more than 20 different interpre- tations of an ambiguous auditory stimulus (repetitions of the word rest) but did not analyze whether certain interpretations were "maintained" or appeared only once. Relevant to this question is the fact that only 3 of the 4 interpretations of Figure 1 seemed to be in the "main rotation" for observers. Future experiments will explore whether manipulating the figure and the conditions of presentation can more convincingly produce a rotation among all 4 interpretations or whether some perceptual limitation makes a rotation of more than 3 interpretations inherently unsustainable. But the fact that the "neglected" interpretation of Figure 1 was also the least common initial interpretation implies that figural aspects of that interpretation, rather than general neural constraints, are responsible for its relative unpopularity.

These results have a potential bearing on whether ambiguous figures are processed holistically or in a piecemeal fashion. Like the theories discussed in the previous paragraph, the well-known satiation theory of multistable figures generally assumes two interpretations. Although this does not seem obligatory, the predominant focus on twostate figures leaves unanswered the question of how successor states are determined. If there are only two states, there are no choices for the successor of a fatigued state. If there are more than two states, the patterns of succession could be essentially random, as though each interpretation was a whole entity, or could follow some organization, as though features could fatigue independent of entire figures; the former possibility is probably more consistent with the original formulation of the satiation hypothesis, but the latter is probably more consistent with the contemporary findings that figures can be perceived, judged, and satiated in a piecemeal fashion (see Hochberg, 1998, for a thorough discussion of this distinction).

The succession patterns of my observers turned out to be partially influenced by expectation. That one of the four interpretations was neglected shows that the observers' behavior was not a simple function of expectation, because nothing in the instructions or conduct of the experiment suggested that any of the four interpretations should be "inferior" to the other three. But considering the three favored interpretations, the observers were influenced by the labels they were given for identifying their interpretations. When the labels and instructions suggested two dimensions of the interpretations, shape and orientation, the observers were sensitive to the same two dimensions and shifted significantly more to a state that shared neither interpretation. But when the labels were arbitrary and the instructions did not mention any organization of the four interpretations, all kinds of shifts were equally likely.

There are some precedents for the fact that ambiguous figure perception is affected by instructions and expectation (e.g., Girgus, Rock, \& Egatz, 1977). My findings also suggest a compromise position between the whole-figure and the figure-quality approaches in that, across the two experiments, neither regime was obligatory. Observers can apparently organize alternative interpretations in a holistic or a featural way, depending on their intentions and expectations. 
Several directions for future research are suggested. First, there may be a way of determining whether a wholefigure or a figural-feature approach to multistable figures is more natural; however, this effort may be futile, since failing to suggest one regime (whole or dimensional) may inherently suggest the other. Second, it should be noted that the sense of piecemeal that is current in ambiguous figure research is not the same as the dimensional approach tested in the present experiments but, rather, concentrates on parts of figures, not on qualities of figures. It may be possible to use the four-state Necker cube to apply this approach to multistable figures. Finally, it would be valuable to test the power of expectations by using instructions that suggest alternative organizations of the four states: Could observers be induced to, say, favor shapepreserving shifts if the states were labeled to suggest that the states with the same shape differ by two dimensions?

\section{REFERENCES}

Attneave, F. (1968). Triangles as ambiguous figures. American Journal of Psychology, 81, 447-453.

Attneave, F. (1971, December). Multistability in perception. Scientific American, 225, 63-71.

Broerse, J., Li, R., \& Ashton, R. (1994). Ambiguous pictorial depth cues and perceptions of nonrigid motion in the three-loop figure. Perception, 23, 1049-1062.

Bucher, N. M., \& Palmer, S. E. (1985). Effects of motion on perceived pointing of ambiguous triangles. Perception \& Psychophysics, 38, 227-236.

Fisher, G. H. (1967). Measuring ambiguity. American Journal of Psychology, 80, 541-557.

Fisher, G. H. (1968). "Mother, father, and daughter": A three-aspect ambiguous figure. American Journal of Psychology, 81, 274-277.

Girgus, J. J., Rock, I., \& Egatz, R. (1977). The effect of knowledge of reversibility on the reversibility of ambiguous figures. Perception \& Psychophysics, 22, 550-556.

Gomez, C., Argandoña, E. D., Solier, R. G., Angulo, J. C., \& VÁzqueZ, M. (1995). Timing and competition in networks representing ambiguous figures. Brain \& Cognition, 29, 103-114.

HochberG, J. A. (1950). Figure-ground reversals as a function of visual satiation. Journal of Experimental Psychology, 40, 682-686.

Hochberg, J. A. (1998). Gestalt theory and its legacy. In J. Hochberg (Ed.), Perception and cognition at century's end (pp. 253-306). San Diego: Academic Press.

Hochberg, J. A., \& Peterson, M. A. (1987). Piecemeal organization and cognitive components in object perception: Perceptually coupled responses to moving objects. Journal of Experimental Psychology: General, 116, 370-380.

Hock, H. S., Schöner, G., \& Voss, A. (1997). The influence of adap- tation and stochastic fluctuations on spontaneous perceptual changes for bistable stimuli. Perception \& Psychophysics, 59, 509-522.

KöHLER, W. (1940). Dynamics in psychology. New York: Liveright. Kubovy, M. (1994). The perceptual organization of dot lattices. Psychonomic Bulletin \& Review, 1, 182-190.

Long, G. M., Toppino, T. C., \& Mondin, G. W. (1992). Prime time: Fatigue and set effects in the perception of reversible figures. Perception \& Psychophysics, 52, 609-616.

Miller, S. M., Liu, G. B., Ngo, T. T., Hooper, G., Riek, S., Carson, R. G., \& Pettigrew, J. D. (2000). Interhemispheric switching mediates perceptual rivalry. Current Biology, 10, 383-392.

PALMER, S. E. (1980). What makes triangles point: Local and global effects in configurations of ambiguous triangles. Cognitive Psychology, 12, 285-305.

Palmer, S. E., \& Bucher, N. M. (1981). Configural effects in perceived pointing of ambiguous triangles. Journal of Experimental Psychology: Human Perception \& Performance, 7, 88-114.

TAY LOR, T. L., \& KLEIN, R. M. (2000). Visual and motor effects in inhibition of return. Journal of Experimental Psychology: Human Perception \& Performance, 26, 1639-1656.

Warren, R M., \& Gregory, R. L. (1958). An auditory analogue of the visual reversible figure. American Journal of Psychology, 71, 612-613.

\section{NOTES}

1. Fisher (1967) actually reported that these figures can be seen seven ways, but that number counts both halves separately for three interpretations in which the figure is seen as two paired components.

2. Six observers in Experiment 1 viewed the figure for $5 \mathrm{~min}$; only the data from the first 4 min were used in the analyses.

3. It is worth emphasizing this point because it may seem that shifts among four states would be skewed by the underrepresentation of one of them. To see why this is not so, assume that diamond-top is never seen at all. The remaining states could shift as follows: stacked-top to stackedbottom (sharing shape), stacked-top to diamond-bottom (nothing shared), stacked-bottom to stacked-top (sharing shape), stacked-bottom to diamond-bottom (sharing orientation), diamond-bottom to stacked-top (nothing shared), and diamond-bottom to stacked-bottom (sharing orientation). Despite the loss of one state, all three shifts are represented by an equal number of cases, and thus, shifts can be treated as independent from visits.

4. The factorial ambiguity of the double-decker Necker may have been anticipated by Attneave (1971). He asserted that a field of scalene triangles has factorial ambiguity, in that the triangles suggest equilateral triangles viewed at a tangent and each of the three orientations of the triangles can be seen on a plane tilted toward or away from the viewer. However, I do not find this effect compelling, and Attneave did not test this phenomenon formally.

5. I am indebted to a reviewer of an earlier version of this paper for pointing out this possibility and the appropriate control condition.

(Manuscript received December 10, 1999; revision accepted for publication June 21, 2001.) 